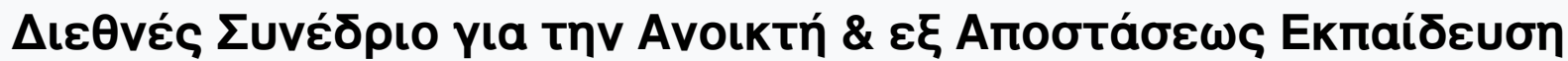

Tóp. 7, Ap. 6B (2013)

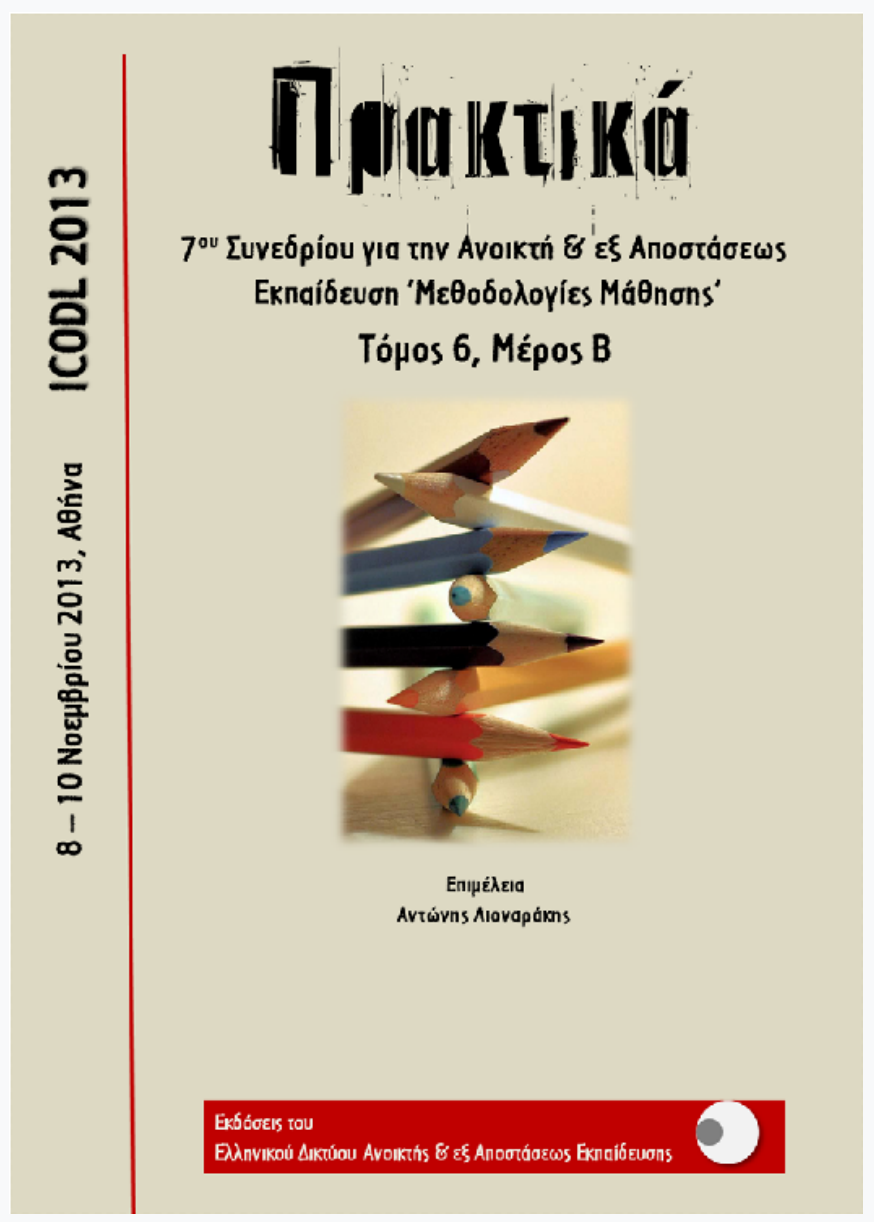

Comparing the camera shot styles of video lectures: Close-up versus broad framing of whiteboard and lecturer

Christina llioudi, Konstantinos Chorianopoulos, Michail N. Giannakos

doi: $\underline{10.12681 / \text { icodl. } 617}$ 


\title{
Comparing the camera shot styles of video lectures: Close-up versus broad framing of whiteboard and lecturer
}

\author{
Christina Ilioudi \\ Department of Informatics \\ Ionian University \\ Corfu, GR-49100 Greece \\ choko@ionio.gr
}

\author{
Konstantinos Chorianopoulos \\ Department of Informatics \\ Ionian University \\ Corfu, GR-49100 Greece
}

\author{
Michail N. Giannakos \\ Department of Computer and \\ Information Science \\ Norwegian University of \\ Science and Technology \\ (NTNU) \\ Trondheim, NO-7491 Norway \\ michailg@idi.ntnu.no
}

\begin{abstract}
Many educational organizations are motivated to create and share instructional videos, but there are no guidelines about the presentation styles. In practice, the presentation style of video lectures ranges from simple video capturing of classroom teaching, up to highly elaborate authoring of video presentations that include closeups and video-cuts of instructors, slides, animations, and interactive drawing boards. In particular, there is limited research about the effects of each presentation style on student learning performance and attitudes. In this work, we examine the effects of video presentation styles in supporting the teaching of mathematics in the secondary education. In addition to a control group that studied through a paper-book, two groups of students attended two distinct styles of video lectures: 1) video capture of class teaching (Talking head style), and 2) close-up video capture of an interactive drawing board with voice-over (Khan style). The participants of our study consisted of 36 students ( 15 boys and 21 girls, 16 years old), who received the respective three treatments (paper book, talking head, khan style), over the course of three math modules in three weeks' time. We found that learning effects show up only after the second week and that the Talking Head style was more effective than the book for complex topics.
\end{abstract}

Keywords: Video, talking head lectures, khan style, satisfaction, playfulness, enjoyment, e-learning, performance

\section{Introduction}

Video lectures have been growing in popularity and many organizations, universities and open learning systems are employing them as a main- or self-study medium, such as Coursera, Udacity, EdX, Khan Academy, TED, and Video Lectures. Although there is a growing interest and use, the benefits and the drawbacks of each different lecture type have not studied yet. The main goal of our research is to explore the effectiveness of different video lectures in teaching mathematics. For this purpose, we produced two different kinds of video lectures: 1) Video capturing of a typical class course and 2) video capturing of an interactive drawing board with voice over. Next, we employed three groups of students, namely two groups for the two kinds of video lecture, as well as one control group. In addition to learning performance 
measurement, we also employed the enjoyment construct, which students reported at the end of their participation.

Research has shown that students benefit from video based or assisted learning (Baloian et al. 2000, Kumar et al. 2001, Piccoli et al. 2001). Specifically nowadays with the growth of many and diverse learning systems like Centra and Matherhorn; the use of video to enhance the learning process attracts much attention. Many educational systems have been developed to use video as the main or secondary tool to enhance the learning process. For example, Carnegie Melon University has created a low cost system called "Just-In-Time Lecture". This system has shown that the use of video in the educational process has analogous results with the traditional classes (Zhang et al. 2006). In the year 2012, there has been a proliferation of Massive Open Online Courses (MOOC), from companies such as Coursera, Udacity, and EdX. Since there is no single standard way or right way of doing a video lecture (Figure 1), it is worth exploring the effects of different styles of video lectures.
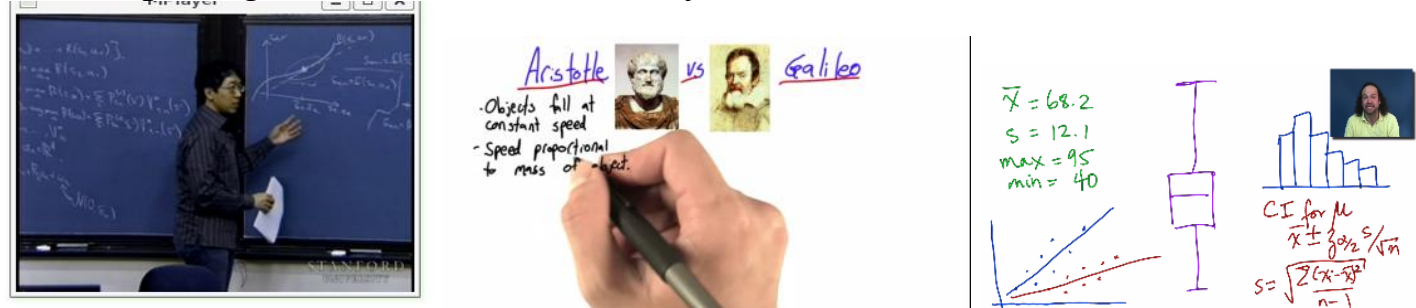

Figure 1. There are many styles of presenting a video lecture. In this work, we focus on the talking head and the interactive drawing board ones which are popular with iTunes University and the Khan Academy respectively

Indeed, videos lectures can take diverse forms and the video lecture style might have effects on important educational parameters such as learning performance and enjoyment. One of the most commonly used is the talking-head lecture, which is the type used by most of the universities (e.g., Stanford, MIT courseware). Another style of video lecture that is growing in popularity is the Khan academy style (hereinafter Khan style). Therefore, the main research goals of our study is to explore the differences among talking head and khan video lectures style, and to compare the differences to the traditional paper book that has been used for centuries during the self-study of the student. The motivation for study is based on the importance of enjoyment derived from a teaching method and is in alignment with previous research (Jiang et al. 2009), who performed a comparison between two teaching methods.

\section{Methodology}

We produced two styles of videos, one with teacher's participation (Talking Head) using the traditional green chalkboard (Figure 2a) and the other one with the teacher's voice over the interactive drawing board (Figure 2b). In our study, the duration of each video was 10 minutes. For the talking head style of video lectures, the content looks like the traditional lecture and it has been a popular video lecture format for many online videos because it is easy to capture and share without going through a resource intensive video editing post-production. In these videos the teacher was presenting a summary of the unit he had taught. For the Khan Style lecture, the viewer concentrates upon the boards' content. Only the teachers' voice and the exercises are participated in this kind of video. The video content focuses on what is being written on the board. The bamboo pen was used for the creation of these videos. This kind of video is very popular in the Khan Academy and Udacity, which was the motivation 
for this study. In both video lecture styles, the teacher was presenting the exact same summary of the module he had taught during the normal course hours.

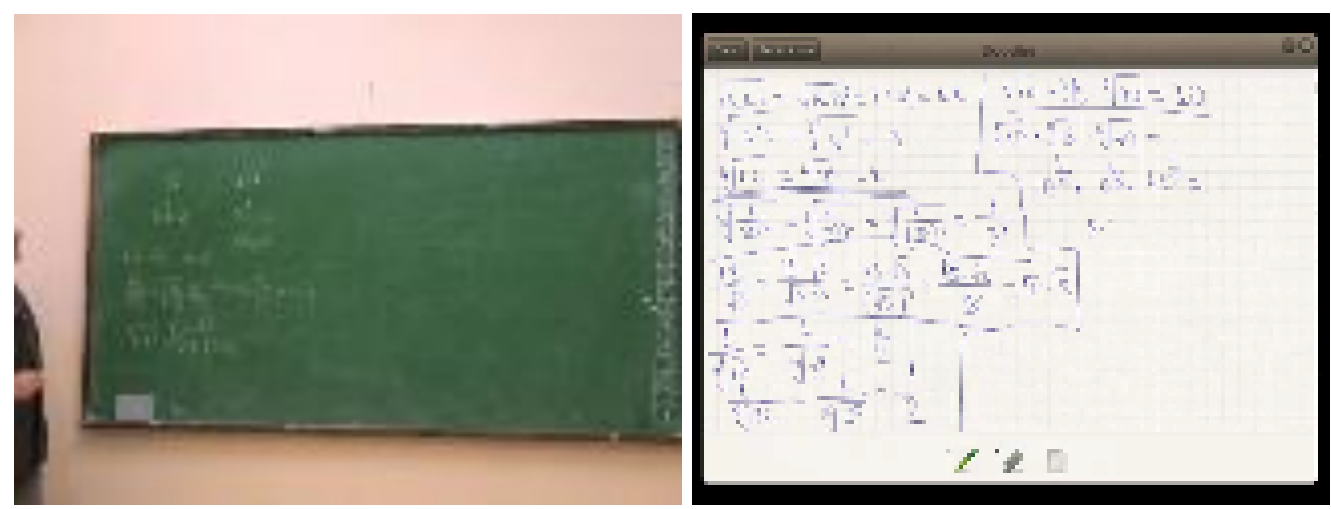

Figure 2 The two styles of video lectures that we employed in the experiment

Thirty-six experimental subjects, 15 boys and 21 girls (16 years old) participated in this study. The students wrote a pre-test on mathematics before the separation into groups, so that the groups are equivalents in terms of previous math performance. After the test we had three groups with 12 students each with a grade average in the pre-test of 16.5 out of 20 points. The presentation of the video took place in the computer laboratory. Every student was watching the 10 minutes video on the computer. One group watched the video with the teacher making a presentation (Talking Head lecture); another group watched the video with a close-up of an interactive board (Khan Style) and the control group browsed through a paper book for the same amount of time.

The study consisted of two parts. In the first part, each one of the groups studied for their module by employing the respective treatments (two styles of video and the paper book). This part of the study was held three times for each group for three different modules of mathematics. Students had ten minutes to watch each style of video and the control group to read the respective module from the book. At the end of each time students solved a test according to the unit they had taught, which lasted twenty minutes. In all cases, the experimental procedure was very strict with regard to the time that the students had. For the implementation of the second part we employed a standard questionnaire which consisted of three questions. The purpose of the questions was to examine the Enjoyment factor (Table 1). Finally, we analysed the data with the use of SPSS program. We used the Mann-Whitney U Test for the processing because of the small sample.

\section{Results}

In the following table we summarize the results of our study.

Table 2. Mean values \& standard deviations of the 3 types

\begin{tabular}{|l|l|l|l|}
\hline & \multicolumn{3}{|c|}{ Mean (S.D.) } \\
\cline { 2 - 4 } & $\begin{array}{l}\text { Talking } \\
\text { Head }\end{array}$ & Khan Style & $\begin{array}{l}\text { Paper } \\
\text { book }\end{array}$ \\
\hline Enjoyment & $2.03(0.96)$ & $1.97(0.82)$ & $2.89(1.15)$ \\
\hline $\begin{array}{l}\text { Learning } \\
\text { Performance 1 }\end{array}$ & $6.38(1.52)$ & $6.31(1.54)$ & $6.26(1.12)$ \\
\hline $\begin{array}{l}\text { Learning } \\
\text { Performance 2 }\end{array}$ & $6.37(2.95)$ & $5.89(2.40)$ & $5.24(3.04)$ \\
\hline $\begin{array}{l}\text { Learning } \\
\text { Performance 3 }\end{array}$ & $7.62(2.70)$ & $6.51(2.05)$ & $4.66(3.50)$ \\
\hline
\end{tabular}


We found that the 'Paper Book' group enjoyed the procedure more than the 'Talking Head' group $(\mathrm{p}<0,05)$. Moreover, we found that there is a siginificant difference in the enjoyment factor between the 'Khan Style' group and 'Paper Book' group. Finally, we used Mann-Whitney U test between the 'Talking

Head' and the 'Khan Style' groups with regard to the learning performance they had during the third module and test in mathematics. We found that the Talking Head group had better performance than the Khan Style group.

Table 3. Testing the differences among Talking Head, Khan Style and Paper book

\begin{tabular}{|c|c|c|c|c|c|c|}
\hline Factor & Type A & Type B & $\mathrm{Z}$ & $\mathrm{U}$ & $\mathrm{P}$ & Result \\
\hline \multirow[t]{3}{*}{ Enjoyment } & Talking Head & Khan Style & & 71 & 0.95 & i.d. \\
\hline & Talking Head & Paper book & -2.07 & 36. & 0.04 & s.d. \\
\hline & Khan Style & Paper book & -2.01 & 37. & 0.04 & s.d. \\
\hline \multirow{3}{*}{$\begin{array}{l}\text { Learning } \\
\text { Performance 1 }\end{array}$} & Talking Head & Khan Style & -0.38 & 65. & 0.71 & i.d. \\
\hline & Talking Head & Paper book & -0.17 & 69 & 0.86 & i.d. \\
\hline & Khan Style & Paper book & 0.00 & 72 & 1.00 & i.d. \\
\hline \multirow{3}{*}{$\begin{array}{l}\text { Learning } \\
\text { Performance } 2\end{array}$} & Talking Head & Khan Style & -0.99 & 55 & 0.32 & i.d. \\
\hline & Talking Head & Paper book & -1.16 & 52 & 0.25 & i.d. \\
\hline & Khan Style & Paper book & -0.52 & 63 & 0.60 & i.d. \\
\hline \multirow{3}{*}{$\begin{array}{l}\text { Learning } \\
\text { Performance } 3\end{array}$} & Talking Head & Khan Style & -1.78 & 41. & 0.08 & i.d. \\
\hline & Talking Head & Paper book & -2.39 & 31 & 0.02 & s.d. \\
\hline & Khan Style & Paper book & -1.48 & 46. & 0.14 & i.d. \\
\hline
\end{tabular}

\section{Conclusion and Further Research}

We found significant statistical differences between the tested video styles and there are also some interesting explanations and useful conclusions. Most notably, the enjoyment measure was reported higher in the control group, who employed a paperbook to study the three modules in mathematics. The familiarity of the students with paper books might be one explanation of enjoying this medium in comparison to the video medium. Another explanation is that students of the Talking Head group and Khan Style group had not employed any video before for their self-study. The preference of video styles might depend on previous exposure to them and there might be cultural and personal parameters, which have to be controlled in further research. In conclusion, further research should pre-test students according to their previous exposure to video lectures and to group them accordingly.

Actual learning performance was slightly improved when the students employed the videos in comparison to the paper book, but this is only after the second week, which indicates that the students need to become familiar with new teaching styles. In particular, there was higher performance in the case of the Talking Head over the Khan Style video lecture. Although we hypothetized that the Khan Style might be result in better performance this was not true. One explanation is that the students felt more familiar with the Talking Head video lecture. Notably, the improvement in 
learning performance was higher for the last mathematics module, which was the most complicated module of the three. Therefore, there might be an influence of the type of course on the learning performance across the self-study mediums and video lectures seem to be superior for complex learning. Moreover, further research should measure the learning performance over more teaching modules than three and over more courses than mathematics.

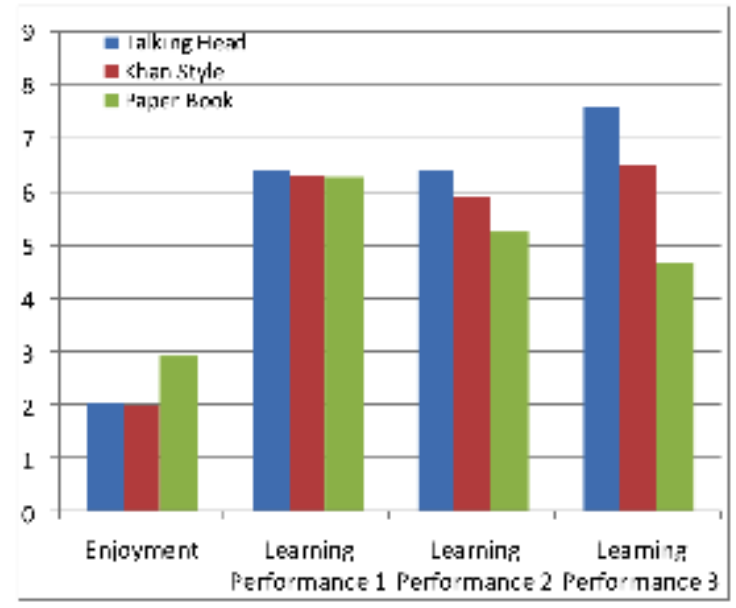

Figure 3. Students enjoyed the book because they had control of browsing, but their performance was higher with the Talking Head video lecture for complicated mathematic module

It is important to note that students watched the videos linearly and did not have the time to watch the video again or reply it. In particular, the use of video did not give the opportunity to students to have any interaction with it when they were watching the video, in contrast to the paper book treatment group, who was observed to browse through the pages. Therefore, in further research, we must allow the students to interact with the videos (Chorianopoulos et al. 2011), but this treatment might need more time than the video length. In practice, allowing students to skip through a video should improve their learning performance, but might come at the cost of additional time. Despite all these limitations all students were positive to employ this way of learning and during the interviews at the end of the research we found that the use of video in self-study motivates the weak students.

\section{Acknowledgements}

The authors would like to express their gratitude to all of the students for volunteering their time. Our very special thanks go to the math teacher for his assistance in our study. We would like also to thank EC project CULT (http://cult.di.ionio.gr/) for providing the equipment.

\section{References}

Baloian, N. A., Pino, J. A., \& Hoppe, H. U. (2000, January). A teaching/learning approach to CSCL. In System Sciences, 2000. Proceedings of the 33rd Annual Hawaii International Conference on (pp. 10-pp). IEEE.

Chorianopoulos, K., Leftheriotis, I., \& Gkonela, C. (2011, June). SocialSkip: pragmatic understanding within web video. In Proceddings of the 9th international interactive conference on Interactive television (pp. 25-28). ACM.

Friedland, G., \& Rojas, R. (2008). Anthropocentric video segmentation for lecture webcasts. Journal on Image and Video Processing, 2008, 9. 
Jiang, J. J., \& Klein, G. (2009). Expectation Confirmation Theory: Capitalizing on Descriptive Power. Handbook of Research on Contemporary Theoretical Models in Information Systems, 384401.

Kumar, A., Kumar, P., \& Basu, S. C. (2001). Student perceptions of virtual education: An exploratory study. In Proceedings of 2001 Information Resources Management Association International Conference (pp. 400-403).

Piccoli, G., Ahmad, R., \& Ives, B. (2001). Web-based virtual learning environments: A research framework and a preliminary assessment of effectiveness in basic IT skills training. MIS quarterly, 401-426.

Signor, L. (2003). An exploration into the reactions of undergraduate students to virtual lectures. Excellence: making the connections, 125

Williams, J., \& Fardon, M. (2007, June). 'Perpetual Connectivity': Lecture Recordings and Portable Media Players. In World Conference on Educational Multimedia, Hypermedia and Telecommunications (Vol. 2007, No. 1, pp. 3083-3091)

Zhang, D., Zhou, L., Briggs, R. O., \& Nunamaker Jr, J. F. (2006). Instructional video in e-learning: Assessing the impact of interactive video on learning effectiveness. Information \& Management, 43(1), 15-27. 